\title{
Genetic control of the stress-sensitivity in hypertensive ISIAH rats
}

\author{
O. Redina ${ }^{1 *}$, S. Smolenskaya ${ }^{1}$, L. Fedoseeva ${ }^{1}$, A. Markel ${ }^{1,2}$ \\ ${ }^{1}$ Institute of Cytology and Genetics SB RAS, Novosibirsk, Russia \\ ${ }^{2}$ Novosibirsk State University, Novosibirsk, Russia \\ *e-mail: oredina@ngs.ru
}

Key words: hypertension, stress, adrenal gland, plasma corticosterone concentration, differentially expressed genes, QTL analysis, ISIAH rat strain

Motivation and Aim: The study of the genetic control of blood pressure and body composition in ISIAH rats being a model of the stress-sensitive hypertension revealed the quantitative trait locus (QTL) on chromosome $\mathrm{X}$ associated with the adrenal gland weight. This locus was also associated with the elevation of blood pressure level and the elevation of the plasma corticosterone concentration under the influence of mild emotional stress [1]. These data suggested that adrenal gland weight may serve as an intermediate phenotype for two other traits related to the enhanced stress-sensitivity of hypertensive ISIAH rats. The current study was directed to the identification of the genes differentially expressed in adrenal glands from hypertensive ISIAH and normotensive WAG rats and localized in the described QTL on Chr. X. The goal of the study was to identify the candidate genes for the elevation of blood pressure level and the elevation of the plasma corticosterone concentration under the influence of mild emotional stress. Methods: The transcriptional profiling (RNA-Seq) of the adrenal glands from ISIAH and WAG male rats was performed to detect the differentially expressed genes (DEGs). Results: The results of RNA-Seq analysis revealed 9 DEGs localized in QTL on Chr. $\mathrm{X}$, which was earlier associated with both the adrenal glands weight and the increase in blood pressure level and plasma corticosterone concentration under the emotional stress. The knowledge-driven filtering of the list of these genes suggested Sms gene encoding spermine synthase as a positional candidate gene, which may be related to the enhanced stress-sensitivity of hypertensive ISIAH rats.

Conclusion: Sms gene encoding spermine synthase may be considered as a positional candidate gene associated with the enhanced stress-sensitivity of hypertensive ISIAH rats. However, the functions of several other discussed DEGs are poorly studied and their further investigation may probably reveal additional candidate genes in the locus. Acknowledgements: Supported by the Budget project No. 0324-2018-0016.

\section{References}

1. Redina O.E. et al. (2013) The genetic control of blood pressure and body composition in rats with stresssensitive hypertension. Clin Exp Hypertens. 35(7):484-95. 